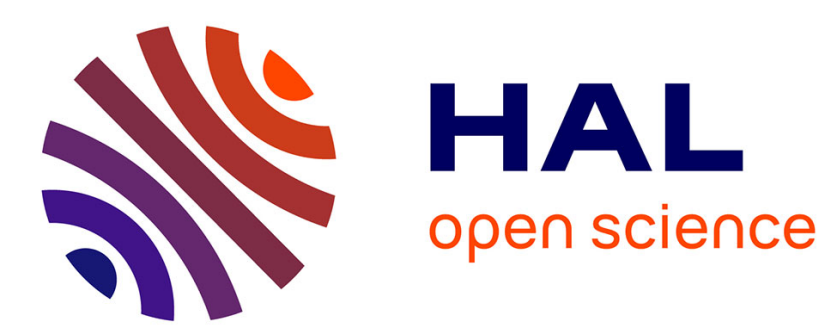

\title{
Fuzzy interpolation by convex completion of sparse rule bases
}

\author{
Laurent Ughetto, Didier Dubois, Henri Prade
}

\section{To cite this version:}

Laurent Ughetto, Didier Dubois, Henri Prade. Fuzzy interpolation by convex completion of sparse rule bases. 9th IEEE International Conference on Fuzzy Systems (FUZZ- IEEE 2000), May 2000, San Antonio (Texas), United States. pp.465-470, 10.1109/FUZZY.2000.838704 . hal-03403393

\section{HAL Id: hal-03403393 \\ https://hal.science/hal-03403393}

Submitted on 27 Oct 2021

HAL is a multi-disciplinary open access archive for the deposit and dissemination of scientific research documents, whether they are published or not. The documents may come from teaching and research institutions in France or abroad, or from public or private research centers.
L'archive ouverte pluridisciplinaire HAL, est destinée au dépôt et à la diffusion de documents scientifiques de niveau recherche, publiés ou non, émanant des établissements d'enseignement et de recherche français ou étrangers, des laboratoires publics ou privés. 


\title{
Fuzzy interpolation by convex completion of sparse rule bases
}

\author{
Laurent UGHeTTO*, Didier DUboIS** and Henri PRADE** \\ * IRIN, 2 rue de la Houssinière, BP 92208, 44322 NANTES Cedex 3, FRANCE - e-mail: ughetto@irin.univ-nantes.fr \\ ** IRIT - CNRS, UPS, 118 route de Narbonne, 31062 TouLOUSE Cedex 4, FRANCE - e-mail: dubois@irit.fr, prade@irit.fr
}

\begin{abstract}
This paper proposes an approach to the interpolation between sparse fuzzy rules, founded on a unique principle which supplements the classical approximate reasoning machinery. The case of rules of the form $A \rightarrow B$, where $A$ and $B$ are intervals is first discussed, and then extended to fuzzy sets.
\end{abstract}

\section{INTRODUCTION}

Fuzzy rule-based systems have been either used as a convenient tool for synthesizing control laws from data, or in a knowledge representation and reasoning perspective in AI. Beside the natural way of representing imprecise and gradual pieces of knowledge that fuzzy rules provide, both applications take advantage of the partial overlapping of their condition parts in the inference mechanism. Indeed, when the input domain is covered by the premises of the rules, wellfounded and established reasoning mechanisms associated to fuzzy rule-based systems have been proposed to generalize the Modus Ponens, as the so-called Compositional Rule of Inference (CRI) introduced by Zadeh in 1973 [1]. By contrast, in case of sparse rules, a special interpolative reasoning mechanism is needed.

Interpolation relies on the assumption of gradual and smooth variation of a variable with respect to another one. This is related to the idea of distance which underlies the natural way human beings fill the gap between extreme rules when they have to cope with an input " $X$ is $A^{\star}$ " which does not fit any of the premises " $X$ is $A_{i}$ " of available sparse rules. Interpolative reasoning naturally subsumes some idea of similarity, where at least two rules of the form "if $X$ is $A_{i}$ then $Y$ is $B_{i}$ " must be used simultaneously. Indeed the result of an interpolation depends on the reference values provided by the rules that are the closest to the input value " $X$ is $A^{\star}$ ". Kóczy and Hirota [2], [3] have proposed some empirical interpolative methods for reasoning from sparse rules, i.e., rules where the $A_{i}$ 's do not achieve a coverage of the input universe $U$, but are rather disjoint and even distant from one another. The problem is then to compute some plausible conclusion " $Y$ is $B^{\star}$ " for an input " $X$ is $A^{\star}$ ", with $B^{\star}$ in between some $B_{i}$ and $B_{j}$, just as $A^{\star}$ is in between the corresponding $A_{i}$ and $A_{j}$, using distances between fuzzy sets. Working on the $\alpha$-cuts $(A)_{\alpha}=[\underline{a}, \bar{a}]$ of fuzzy sets $A$, a natural idea is to compute the $\alpha$-cut of $B^{\star}$ as $\left[\underline{b}^{\star}, \bar{b}^{\star}\right]=\left[\lambda \cdot \underline{b}_{i}+(1-\lambda) \cdot \underline{b}_{j}, \lambda^{\prime} \cdot \bar{b}_{i}+\left(1-\lambda^{\prime}\right) \cdot \bar{b}_{j}\right]$ on the basis of the solutions $\lambda$ and $\lambda^{\prime}$ of the equations $\underline{a}^{\star}=\lambda \cdot \underline{a}_{i}+(1-\lambda) \cdot \underline{a}_{j}$ and $\bar{a}^{\star}=\lambda^{\prime} \cdot \bar{a}_{i}+\left(1-\lambda^{\prime}\right) \cdot \bar{a}_{j}$ where $\left[\underline{a}^{\star}, \bar{a}^{\star}\right]$ is the $\alpha$-cut of $A^{\star}$. Unfortunately, the $\alpha$-cuts of such a $B^{\star}$ may not be nested and we may even have $\underline{b}^{\star}>\vec{b}^{\star}$. See Yan et al. [4], Qiao et al. [5], Hsiao et al. [6] for further developments along this line.
This interpolation problem can also be formalized as the . computation of a convex sum of $B_{i}$ and $B_{j}$ based on a parameter $\lambda$ imprecisely constrained by $u_{0}=\lambda \cdot u_{1}+(1-\lambda) \cdot u_{2}$, with $u_{0}$ restricted by $A^{\star}, u_{1}$ by $A_{i}$ and $u_{2}$ by $A_{j}$ [7], [8]. However, while the above-mentioned drawback is avoided, the result of the inference may be very imprecise.

A general method has been proposed by Bouchon-Meunier et al. [9], [10] on the basis of the concepts of location and shape of a fuzzy set and the evaluation of the distinguishability of two fuzzy sets with regard to these concepts and in agreement with the analogical scheme proposed in [11]. More precisely, if $A^{\star}$ is between $A_{i}$ and $A_{i+1}$, with respect to some order on fuzzy sets, a conclusion $B^{\star}$ regarding variable $Y$ is constructed between $B_{i}$ and $B_{i+1}$, in such a way that the location and shape distinguishabilities of $B^{\star}$ and $B_{i}$, of $B^{\star}$ and $B_{i+1}$, are analogous to the location and shape distinguishabilities of $A^{\star}$ and $A_{i}$, of $A^{\star}$ and $A_{i+1}$. However, several degrees of freedom remain in this rather general scheme.

In this paper, the proposed approach relies on a unique linear interpolation principle which, once accepted, fully determines the result of the inference. The underlying idea of the approach, first briefly suggested at the end of [12], and closely related to some mechanism first partially described in [13] is the following. In classical reasoning, the two rules $\left(A \cap A^{\prime}\right) \rightarrow\left(B \cap B^{\prime}\right)$ and $\left(A \cup A^{\prime}\right) \rightarrow\left(B \cup B^{\prime}\right)$ are direct consequences of the set of rules $\left\{A \rightarrow B, A^{\prime} \rightarrow B^{\prime}\right\}$. The basic principle of the proposed approach is to augment this machinery on the real line, by assuming that it is also allowed to derive from the two rules any new rule $A_{\lambda} \rightarrow B_{\lambda}$, where $\lambda \in[0,1]$, and where $A_{\lambda}=\lambda \cdot A+(1-\lambda) \cdot A^{\prime}$ and $B_{\lambda}=\lambda \cdot B+(1-\lambda) \cdot B^{\prime}$, obtained by interval arithmetics.

Considering two sparse rules $A_{i} \rightarrow B_{i}$ and $A_{i+1} \rightarrow B_{i+1}$, and an observation $A^{\star}$ in between $A_{i}$ and $A_{i+1}$, the method consists in computing a union or an intersection of several $A_{\lambda}$ which best fit $A^{\star}$. Then, the union or intersection of the corresponding $B_{\lambda}$ gives the corresponding output $B^{\star}$, according to a fuzzy linear interpolation scheme between the two initial rules, as it will be shown.

In Section II, this paper first studies linear interpolative reasoning between two non fuzzy rules, i.e., rules of the form $A \rightarrow B$, where $A$ and $B$ are intervals. The case of one input and one output rules is first considered, followed by a brief discussion of interpolation with multiple input rules. Then, in Section III the results are extended to fuzzy rules, by approximating $A^{\star}$ with a union or an intersection of $A_{\lambda} \mathrm{s}$ (and then $B^{\star}$ with $\left.B_{\lambda} s\right)$. 


\section{CASE OF NON-FUZZY RULES}

This section deals with interpolative reasoning between the two non-fuzzy sparse rules of the set $\mathcal{K}=\left\{A_{0} \rightarrow B_{0}, A_{1} \rightarrow\right.$ $\left.B_{1}\right\}$. The operator $\rightarrow$ is then the classical logic implication. The rules in $\mathcal{K}$ being sparse (or disjoint) means that $A_{0} \cap A_{1}=$ $\emptyset$. In this section, as only intervals (instead of fuzzy sets) are considered, an interval $A$ is denoted $[\underline{a}, \bar{a}]$.

Now, consider a piece of information $A^{\star}$ (e.g., an observation) on the input variable $X$. The set $A^{\star}$ is also supposed to be an interval, which lies in between $A_{0}$ and $A_{1}$, on the input universe $U$. It encompasses the case where $A^{\star}$ somewhat overlaps with $A_{0}$ and/or $A_{1}$, but still being in between (i.e., $\left.\underline{a}_{0} \leq \underline{a}^{\star} \leq \bar{a}^{\star} \leq \bar{a}_{1}\right)$, as the interpolation mechanism also has influence on $A_{0}$ and $A_{1}$, as shown on Figure 5.c.

The problem is then to determine an interval $B^{\star}$ in between $B_{0}$ and $B_{1}$ (which restricts the possible values for the variable $Y$, on the output universe $V$ ), such that $A^{\star} \rightarrow B^{\star}$ is a valid rule w.r.t. $A_{0} \rightarrow B_{0}$ and $A_{1} \rightarrow B_{1}$ according to the principle proposed in the introduction.

For the sake of simplicity, the case of one input and one output rules is first detailed. Moreover, in all this section, only the increasing case is considered, i.e., the case where $A_{1}$ is "greater" than $A_{0}$, and $B_{1}$ is "greater" than $B_{0}$ (see for instance Figure 1). All the results obtained in this case can be easily carried over to the decreasing case.

\section{A. One input, one output rules}

In this first case, the premises of the two rules in $\mathcal{K}$, namely $A_{0}$ and $A_{1}$, as well as their conclusions $B_{0}$ and $B_{1}$, are compact subsets of the real line $\mathbb{R}$.

Assuming a linear interpolation model between the two rules in $\mathcal{K}$ leads to the family of rules $A_{\lambda} \rightarrow B_{\lambda}$, for all $\lambda \in[0,1]$, where $A_{\lambda}=\lambda \cdot A_{1}+(1-\lambda) \cdot A_{0}$ and $B_{\lambda}=$ $\lambda \cdot B_{1}+(1-\lambda) \cdot B_{0}$. An example of such an $A_{\lambda}$ and a $B_{\lambda}$, with $\lambda=0.5$, is shown on Figure 1 .

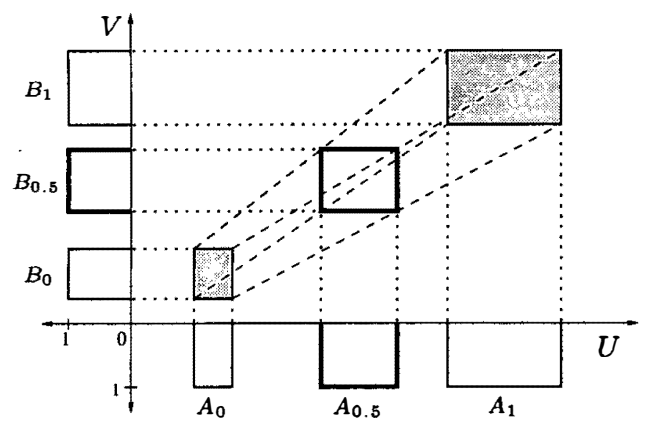

Fig. 1. Linear interpolation between two non fuzzy rules

Thus, given that $A_{\lambda} \rightarrow B_{\lambda}$ are the rules obtained from $\mathcal{K}$ by linear interpolation, the idea is to compute a union or an intersection of $A_{\lambda}$ s that corresponds to (or best fits) $A^{\star}$. Then, the corresponding union or intersection of $B_{\lambda}$ s gives the output interval $B^{\star}$. This idea comes from the fact that the two rules $\left(A \cap A^{\prime}\right) \rightarrow\left(B \cap B^{\prime}\right)$ and $\left(A \cup A^{\prime}\right) \rightarrow\left(B \cup B^{\prime}\right)$ are logical consequences of a set of two rules $\left\{A \rightarrow B, A^{\prime} \rightarrow B^{\prime}\right\}$, and the generalization of this property to a set of more than 2 rules.

Then, given the set of two rules $\mathcal{K}$, and an observation $A^{\star}$ in between the premises of the rules, three different situations can occur:

1. $A^{\star}=A_{\lambda}$ for some $\lambda \in[0,1]$. Then, the rule $A_{\lambda} \rightarrow B_{\lambda}$ obtained by linear interpolation directly gives the conclusion $B^{\star}=B_{\lambda}$.

2. $A^{\star} \supset A_{\lambda}$ for some $\lambda \in[0,1]$. Then, with $\mathcal{S}_{\max }=$ $\left\{\lambda, A^{\star} \supset A_{\lambda}\right\} \subseteq[0,1]$, the set $A^{\star}$ is obtained by:

$$
\bigcup_{\lambda \in \mathcal{S}_{\max }} A_{\lambda}=A^{\star}, \text { and then } B^{\star}=\bigcup_{\lambda \in \mathcal{S}_{\max }} B_{\lambda} \text {. }
$$

3. $A^{\star} \subset A_{\lambda}$ for some $\lambda \in[0,1]$. Then, with $\mathcal{S}_{\max }=$ $\left\{\lambda, A^{\star} \subset A_{\lambda}\right\} \subseteq[0,1]$, the set $A^{\star}$ is obtained by:

$$
\bigcap_{\lambda \in \mathcal{S}_{\max }} A_{\lambda}=A^{\star}, \quad \text { and then } \quad B^{\star}=\bigcap_{\lambda \in \mathcal{S}_{\max }} B_{\lambda} \text {. }
$$

Clearly, only the last two cases need to be detailed.

Proposition 1: These three cases are mutually exclusive.

Proof: As previously said, each interval $A$ is denoted by its lower and upper bounds $[\underline{a}, \bar{a}]$. The values $\underline{\lambda}$ and $\bar{\lambda}$ given by:

- $\underline{a}^{\star}=\underline{\bar{\lambda}} \cdot \underline{a}_{1}+(1-\underline{\bar{\lambda}}) \cdot \underline{a}_{0}$, and then: $\underline{\bar{\lambda}}=\left(\underline{a}^{\star}-\underline{a}_{0}\right) /\left(\underline{a}_{1}-\underline{a}_{0}\right)$, - $\bar{a}^{\star}=\bar{\lambda} \cdot \bar{a}_{1}+(1-\overline{\bar{\lambda}}) \cdot \bar{a}_{0}$, and then: $\overline{\bar{\lambda}}=\left(\bar{a}^{\star}-\bar{a}_{0}\right) /\left(\bar{a}_{1}-\bar{a}_{0}\right)$, are such that the left side of $A_{\underline{\lambda}}$ matches the left side of $A^{\star}$, and the right side of $A_{\bar{\lambda}}$ matches the right side of $A^{\star}$. Then, from the values $\underline{\lambda}$ and $\bar{\lambda}$, three cases can occur (see Figure 2):

- $\underline{\lambda}=\bar{\lambda}$ corresponds to the first case, as $A_{\underline{\lambda}}=A_{\bar{\lambda}}=A^{\star}$,

- $\underline{\lambda}<\bar{\lambda}$ means that $A_{\underline{\lambda}} \subset A^{\star}$ and $A_{\bar{\lambda}} \subset A^{\star}$ (case number 2), - $\underline{\lambda}>\bar{\lambda}$ means that $A_{\underline{\lambda}} \supset A^{\star}$ and $A_{\bar{\lambda}} \supset A^{\star}$ (case number 3 ), and these three cases are obviously exclusive.

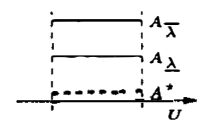
Case 1: $\begin{gathered}A^{\star}=A_{\lambda} \\ \qquad \lambda=\bar{\lambda}\end{gathered}$

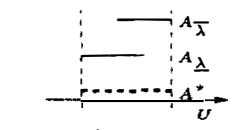

Case 2: $A^{*}$ bigger than $A_{\lambda}$ $\underline{\lambda}<\bar{\lambda}$

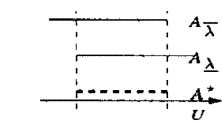
Case 3: $A^{*} \underset{\substack{\text { smaller than } \\ \lambda}}{>} A_{\lambda}$ $\lambda>\bar{\lambda}$
Fig. 2. The 3 cases depending on $\underline{\lambda}$ and $\bar{\lambda}$

Now, since the method can be viewed as the replacement of the initial set of two rules $\mathcal{K}=\left\{A_{0} \rightarrow B_{0}, A_{1} \rightarrow B_{1}\right\}$, with the (infinite) set of rules $\mathcal{L}=\left\{A_{\lambda} \rightarrow B_{\lambda}, \lambda \in[0,1]\right\}$ (obtained from the two rules in $\mathcal{K}$ ), the following proposition gives a fundamental result.

Proposition 2: The set of rules $\mathcal{L}=\left\{A_{\lambda} \rightarrow B_{\lambda}, \lambda \in[0,1]\right\}$, obtained by linear interpolation from $\mathcal{K}=\left\{A_{0} \rightarrow B_{0}, A_{1} \rightarrow\right.$ $\left.B_{1}\right\}$, may be potentially incoherent, in the sense of coherence given in [14]. It means that two rules in $\mathcal{L}$ can be simultaneously triggered (i.e., $A_{\lambda} \cap A_{\lambda^{\prime}} \neq \emptyset$ ), while they give incompatible conclusions (i.e., $B_{\lambda} \cap B_{\lambda^{\prime}}=\emptyset$ ).

Proof: See an example of coherence and incoherence cases on Figure 3 and a characterization of the incoherence cases given by Proposition 8 .

It is clear from the definition that the incoherence can be especially detected in the third case, when $\bigcap_{\lambda \in S} A_{\lambda}=A^{\star}(\neq \emptyset)$ and $\bigcap_{\lambda \in \mathcal{S}} B_{\lambda}=\emptyset$ in (2). 
As a consequence, when $\mathcal{L}$ is a potentially incoherent set of rules, intersections of $A_{\lambda}$ s (i.e., case 3 ) shouldn't be used. A more cautious approach could even consider that the interpolative inference method proposed here may not be used when $\mathcal{L}$ is not coherent. Indeed, it will be shown in the following that this method is equivalent to the CRI applied to $\mathcal{L}$ only when $\mathcal{L}$ is coherent. Thus, for incoherent cases, a slightly different method will be proposed.

\section{B. Case of $A^{\star} s$ bigger than $A_{\lambda} s$}

First consider the case of a large $A^{\star}$, i.e., an interval such that $A^{\star} \supset A_{\lambda}$ for some $\lambda \in[0,1]$.

Proposition 3: Given $A^{\star}$ such that $A^{\star} \supset A_{\lambda}$, for one $\lambda \in$ $[0,1]$, the set $\mathcal{S}_{\max }=\left\{\lambda, A_{\lambda} \supset A^{\star}\right\}$ is the maximal (for inclusion) subset of $[0,1]$ which verifies (1). This set $S_{\max }$ is the interval $[\underline{\lambda}, \bar{\lambda}]$, where $\underline{\lambda}=\left(\underline{a}^{\star}-\underline{a}_{0}\right) /\left(\underline{a}_{1}-\underline{a}_{0}\right)$ and $\bar{\lambda}=$ $\left(\bar{a}^{\star}-\bar{a}_{0}\right) /\left(\bar{a}_{1}-\bar{a}_{0}\right)$.

Proof: Clearly, $\underline{\lambda}$ (resp. $\bar{\lambda}$ ) is the smallest (resp. the greatest) $\lambda$ such that $A_{\lambda} \supset A^{\star}$. Then, it can be easily shown that $\forall \lambda \in$ $[\underline{\lambda}, \bar{\lambda}], A_{\lambda} \supset A^{\star}$. Thus, $\mathcal{S}_{\max }=[\underline{\underline{\lambda}}, \bar{\lambda}]$, and this set is maximal for inclusion.

This proposition gives one possible set $\mathcal{S}$ which verifies (1), and thus such that $B^{\star}=\bigcup_{\lambda \in \mathcal{S}} B_{\lambda}$. However, this set does not really enable the computation of $B^{\star}$, since it is an infinite subset of $[0,1]$.

A first way to solve this problem could consist in computing a finite subset $\mathcal{S}$ of $\mathcal{S}_{\max }$ such that $A^{\star}=\bigcup_{\lambda \in \mathcal{S}} A_{\lambda}$. However, as one can get a set $\mathcal{S}$ such that $\bigcup_{\lambda \in \mathcal{S}} A_{\lambda}=A^{\star}$ and $\bigcup_{\lambda \in \mathcal{S}} B_{\lambda} \neq B^{\star}$ (with $A^{\star}$ and $B^{\star}$ defined by (1)) because of lack of convexity of $\bigcup_{\lambda \in \mathcal{S}} B_{\lambda}$, this approach is not developed here.

Another approach is based on the fact that $B^{\star}$ is an interval on the continuous universe $V$, namely $\left[\underline{b}^{\star}, \vec{b}^{\star}\right]$. Thus, in order to define $B^{\star}$, it is sufficient to compute its two boundaries $\underline{b}^{\star}$ and $\vec{b}^{\star}$.

Proposition 4: Given the two sparse rules in $\mathcal{K}$, and an observation $A^{\star}$ in between $A_{0}$ and $A_{1}$, such that $A^{\star} \supset A_{\lambda}$, for one $\lambda \in[0,1]$, the corresponding output $B^{\star}$ is given by $\left[\underline{b}^{\star}, \vec{b}^{\star}\right]$, with $\underline{b}^{\star}=\underline{b}_{\underline{\lambda}}$ and $\bar{b}^{\star}=\bar{b}_{\bar{\lambda}}$, or equivalently:

$B^{\star}=\left[\frac{\underline{b}_{1}-\underline{b}_{0}}{\underline{a}_{1}-\underline{a}_{0}} \underline{a}^{\star}+\frac{\underline{a}_{1} \underline{b}_{0}-\underline{a}_{0} \underline{b}_{1}}{\underline{a}_{1}-\underline{a}_{0}}, \frac{\bar{b}_{1}-\bar{b}_{0}}{\bar{a}_{1}-\bar{a}_{0}} \bar{a}^{\star}+\frac{\bar{a}_{1} \bar{b}_{0}-\bar{a}_{0} \bar{b}_{1}}{\bar{a}_{1}-\bar{a}_{0}}\right]$ Proof: The set $B^{\star}$ is given by the interval $\bigcup_{\lambda \in \mathcal{S}_{\max }} B_{\lambda}$, and $\mathcal{S}_{\max }$ is the interval $[\underline{\lambda}, \bar{\lambda}]$. The lower bound of $B^{\star}$ is then the smallest value of the lower bounds of the $B_{\lambda} \mathrm{s}$, for $\lambda \in \mathcal{S}_{\max }$. And it is clear that this value is the lower bound of $B_{\lambda}$. Thus $\underline{b}^{\star}=\underline{b}_{\underline{\lambda}}$. The proof is the same for the upper bound.

\section{Case of $A^{\star} s$ smaller than $A_{\lambda} s$}

Now consider the third case, where $A^{\star}$ is included in one $A_{\lambda}$. The next proposition is the counterpart of Proposition 3 for small $A^{\star} \mathrm{s}$ (the same notations are used).

Proposition 5: Given $A^{\star}$ such that $A^{\star} \subset A_{\lambda_{0}}$, for one $\lambda_{0} \in$ $[0,1]$, the set $\mathcal{S}_{\max }=\left\{\lambda, A_{\lambda} \subset A^{\star}\right\}$ is the maximal (for inclusion) subset of $[0,1]$ which verifies (2). This set $\mathcal{S}_{\max }$ is the interval $[\bar{\lambda}, \underline{\lambda}]$, where $\underline{\lambda}=\left(\underline{a}_{1}-\underline{a}^{\star}\right) /\left(\underline{a}_{1}-\underline{a}_{0}\right)$ and $\bar{\lambda}=$ $\left(\bar{a}_{1}-\bar{a}^{\star}\right) /\left(\bar{a}_{1}-\bar{a}_{0}\right)$.

Proof: It is similar to the proof of Proposition 3.

As in section II-B, the set $\mathcal{S}_{\min }$ is infinite, and is not very useful for the computation of $B^{\star}$. But in case of $A^{\star} s$ smaller than $A_{\lambda} \mathrm{s}$, only two values in $\mathcal{S}$ are necessary to obtain $A^{\star}$. Indeed, $A^{\star}=A_{\underline{\lambda}} \cap A_{\bar{\lambda}}$, as shown on Figure 2 .

Proposition 6: The set $\mathcal{S}_{\min }=\{\bar{\lambda}, \underline{\lambda}\}$ is the minimal (for inclusion) set $\mathcal{S}$ which verifies (2).

Proof: It is clear that $\mathcal{S}_{\min }$ verifies (2). The set $\mathcal{S}_{\min }$ is minimal since the suppression of one of its elements leads to a set which clearly do not verify (2). And it is the minimal set since the replacement of one element in $\mathcal{S}_{\min }$ also leads to a set which do not verify (2), as clear from Figure 2.

Here, the set $B^{\star}$ can be computed as

$$
B^{\star}=\bigcap_{\lambda \in \mathcal{S}_{\text {rnin }}} B_{\lambda}=B_{\bar{\lambda}} \cap B_{\underline{\lambda}} .
$$

An equivalent way to compute $B^{\star}$ is given by the counterpart of Proposition 4 for $A^{\star}$ s smaller than $A_{\lambda} \mathrm{s}$.

Proposition 7: Given the two sparse rules in $\mathcal{K}$, and an observation $A^{\star}$ in between $A_{0}$ and $A_{1}$, such that $A^{\star} \subset A_{\lambda}$, for one $\lambda \in[0,1]$, the corresponding output $B^{\star}$ is given, when it exists, by $\left[\underline{b}^{\star}, \bar{b}^{\star}\right]$, with $\underline{b}^{\star}=\underline{b}_{\underline{\lambda}}$ and $\bar{b}^{\star}=\bar{b}_{\bar{\lambda}}$, or equivalently: $B^{\star}=\left[\frac{\underline{b}_{1}-\underline{b}_{0}}{\underline{a}_{1}-\underline{a}_{0}} \underline{a}^{\star}+\frac{\underline{a}_{1} \underline{b}_{0}-\underline{a}_{0} \underline{b}_{1}}{\underline{a}_{1}-\underline{a}_{0}}, \frac{\bar{b}_{1}-\bar{b}_{0}}{\bar{a}_{1}-\bar{a}_{0}} \bar{a}^{\star}+\frac{\bar{a}_{1} \bar{b}_{0}-\bar{a}_{0} \bar{b}_{1}}{\bar{a}_{1}-\bar{a}_{0}}\right]$. Proof: It comes straightforwardly from the computation of the intersection $B_{\bar{\lambda}} \cap B_{\underline{\lambda}}=\left[\underline{b}_{\lambda}, \bar{b} \bar{\lambda}\right]$, since $\underline{\lambda}>\bar{\lambda}$.

Clearly, the expression of $B^{\star}$ is the same in Propositions 4 and 7. However, in this case, the interval $B^{\star}$ does not always exist.

\section{Detection of an incoherent situation}

The two rules $A_{\underline{\lambda}} \rightarrow B_{\underline{\lambda}}$ and $A_{\bar{\lambda}} \rightarrow B_{\bar{\lambda}}$ are said to be incoherent when $A^{\star}=A_{\underline{\lambda}} \cap A_{\bar{\lambda}} \neq \emptyset$ and $B^{\star}=B_{\underline{\lambda}} \cap B_{\bar{\lambda}}=\emptyset$.

These cases of incoherence depend on properties on the antecedent $\left(A_{0}\right.$ and $\left.A_{1}\right)$ and conclusion parts $\left(B_{0}\right.$ and $\left.B_{1}\right)$ of the two rules in $\mathcal{K}$. Thus, it is possible to determine a necessary and sufficient coherence condition for the set $\mathcal{L}$.

Proposition 8: Let $\underline{\Gamma}$ be the line defined by the two points $\left(\underline{a}_{0}, \underline{b}_{0}\right)$ and $\left(\underline{a}_{1}, \underline{b}_{1}\right)$, and $\bar{\Gamma}$ the line defined by the two points $\left(\bar{a}_{0}, \bar{b}_{0}\right)$ and $\left(\bar{a}_{1}, \bar{b}_{1}\right)$, as shown on Figure 3 . The set $\mathcal{L}$ is potentially incoherent if the line $\underline{\Gamma}$ is above the line $\bar{\Gamma}$ for at least one point in $\left[\bar{a}_{0}, \underline{a}_{1}\right]$, i.e., if $\exists u \in\left[\bar{a}_{0}, \underline{a}_{1}\right], \underline{\Gamma}(u)>\bar{\Gamma}(u)$. Proof: Consider a value $u \in U$ such that $\underline{\Gamma}(u)>\bar{\Gamma}(u)$, and $A^{\star}=\{u\}$. The computation of $B_{\underline{\lambda}}$ and $B_{\bar{\lambda}}$ (as defined in Section II-C) shows that $B_{\underline{\lambda}}=\left[\underline{\Gamma}(u), \overline{\bar{b}}_{\underline{\lambda}}\right]$ and that $B_{\bar{\lambda}}=\left[\underline{b}_{\bar{\lambda}}, \bar{\Gamma}(u)\right]$. And thus $B^{\star}=B_{\underline{\lambda}} \cap B_{\bar{\lambda}}=\emptyset($ since $\underline{\Gamma}(u)>\bar{\Gamma}(u))$, which means incoherence.

Note that when the two lines $\underline{\Gamma}$ and $\bar{\Gamma}$ intersect, $\mathcal{L}$ is potentially incoherent on a subset of $\left[\bar{a}_{0}, \underline{a}_{1}\right]$ only. As a corollary of Proposition 8 , one can compute the interval of potential incoherence in this case, which corresponds to the subinterval of $\left[\bar{a}_{0}, \underline{a}_{1}\right]$ for which $\underline{\Gamma}$ is above $\bar{\Gamma}$.

Beside the calculations, it is important to understand the meaning of potential incoherence for sparse rules. Indeed, 

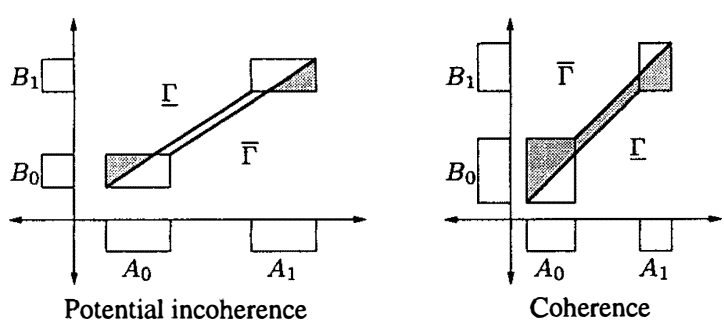

Fig. 3. Coherence and potential incoherence cases

considering the CRI (as a generalization of the Modus Ponens) for the reasoning mechanism, it has been shown that sparse rules cannot lead to incoherence (see [14]). Strictly speaking, $\mathcal{K}$ is never incoherent. Only $\mathcal{L}$ may be incoherent. However, when $\mathcal{L}$ is incoherent, $\mathcal{K}$ can be said incoherent w.r.t. the interpolative inference mechanism proposed here, according to a generalization of the notion of coherence for rule-based systems: "for any allowed input, the system must give a nonempty output".

From the equations, it can be seen that potential incoherence may occur either when the $B \mathrm{~s}$ are sparser than the $A \mathrm{~s}$, or when the $B \mathrm{~s}$ are more precise than the $A$ s (or a combination of these two effects since they can compensate). The $B$ s being sparser than the $A$ s means that they are divergent, as the conclusion parts are less similar than the condition parts of the rules. It seems a natural extension of the notion of incoherence from a similarity point of view (see Figure 4 ). Thus, $\mathcal{K}$ can be said potentially incoherent if two rules in $\mathcal{L}$ are potentially incoherent in the sense of [14], as shown on Figure 4.b for $A_{0.4} \rightarrow B_{0.4}$ and $A_{0.6} \rightarrow B_{0.6}$.

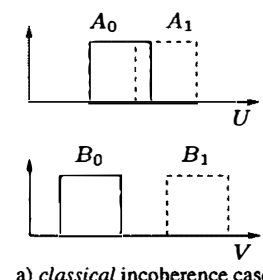

a) classical incoherence case
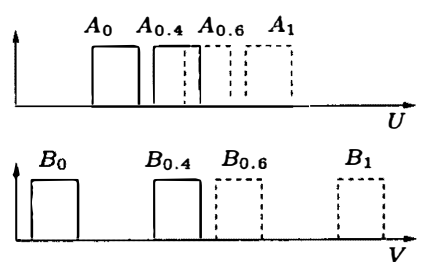

b) generalized incoherence case
Fig. 4. Generalized notion of incoherence

Moreover, in this special case of incoherence, applying (2) with a well-chosen interval $A^{\star}$ could lead to a singleton for $B^{\star}$. In this particular case, obtaining a singleton, i.e., a precise value for $B^{\star}$, from an imprecise observation $A^{\star}$ and two (even more) imprecise rules seems hardly acceptable. In such a case, if $A^{\star}$ would be made still more precise, $B^{\star}$ becomes empty. By contrast, it can be shown that $B^{\star}$ s obtained in the coherent case are always more imprecise than the corresponding $A^{\star}$. This is why this method, and especially the intersection of rules shouldn't be used in the incoherent case.

\section{E. Comparisons and an inference algorithm based on the $C R I$}

It is interesting to compare this interpolative reasoning method with previously proposed ones, and especially the two ones in [15], shown on Figure 5.a-b. These two methods are similar to the one proposed in this paper (for coherence cases) since they are all equivalent to the definition of a relation on the Cartesian product of the input output universes $U \times V$ in between the rules, and the application of the CRI as the reasoning mechanism.

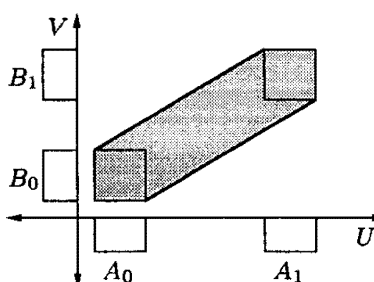

a) rules as imprecise points

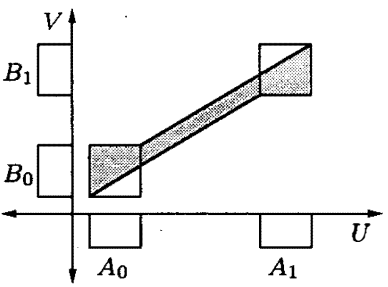

c) new method (coherent case)

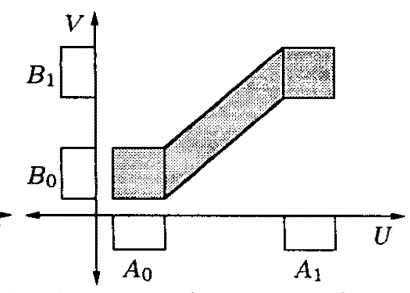

b) rules as parts of an imprecise function
Fig. 5. Three different interpolative mechanisms

From a semantic point of view, the first method considers the pair $(A, B)$ (from the rule $A \rightarrow B$ ), as a fuzzy point and applies the extension principle (see [15], [8]). It means that the relation is the set of all segments of the form $\left[m_{0}, m_{1}\right]$, where $m_{0}$ is a point in $A_{0} \times B_{0}$, and $m_{1}$ a point in $A_{1} \times B_{1}$. The obtained relation is the convex hull of $\left(A_{0} \times B_{0}\right) \cup\left(A_{1} \times B_{1}\right)$.

The second method considers the pair $(A, B)$ as a part of a fuzzy function from $U$ to $V$. Thus, for a point $(u, v) \in U \times V$, if $u \in A$, then $v \in B$. The interpolation between these two rules only considers the right side of $A_{0}$ and the left side of $A_{1}$, and takes the corresponding convex hull. It leads to the relation depicted on Figure 5.b. Although this relation is more restrictive than the previous one, it is more in accordance with the notion of rule. Indeed, if $u \in A$, then $v$ cannot be outside $B$, while it is the case in Figure 5.a (see the grey region above the rectangle representing $A_{0} \times B_{0}$ on $U \times V$ ).

The relations defined by these interpolation processes can be represented by two bounding lines. It is also the case with the third figure (5.c), which corresponds to the method proposed here in a coherent case. It can be represented by the two lines $\Gamma$ and $\bar{\Gamma}$ defined above, and corresponds to the relation $\bigcap_{\lambda \in[0,1]} A_{\lambda} \rightarrow B_{\lambda}$, defined by the set of rules $\mathcal{L}$. This relation is more restrictive than the two others.

In these three cases, the reasoning mechanism can be the CRI applied to the relation defined on $U \times V$ by two bounding lines, as shown on Figure 5. Thus, it comes down to the evaluation of a function in two points. For the proposed method, it is $B^{\star}=\left[\underline{\Gamma}\left(\underline{a}^{\star}\right), \bar{\Gamma}\left(\bar{a}^{\star}\right)\right]$ for the case of an increasing interpolative function (as on Figure 5.c). This result is in accordance with $B^{\star}=A^{\star} \circ \bigcap_{\lambda \in[0,1]} A_{\lambda} \rightarrow B_{\lambda}$, i.e., with the CRI. 
In the incoherent case, the CRI cannot be applied, since the relation represented by the set of rules $\mathcal{L}$ on $U \times V$ is empty. However, if the entire set $\mathcal{L}$ is incoherent in this case, considering only one rule from $\mathcal{L}$ still makes sense, as well as considering one rule, obtained by union of rules in $\mathcal{L}$. Indeed, a rule $A_{\lambda} \rightarrow B_{\lambda}$, for one $\lambda \in[0,1]$ such that $A_{\lambda}$ overlap neither $A_{0}$, nor $A_{1}$ is always coherent w.r.t. $\mathcal{K}$. And it is clear that the case 1 of Section II-A (for which $\exists \lambda \in[0,1]$ such that $A^{\star}=A_{\lambda}$ ) corresponds to the CRI with $A^{\star}$ and such a rule. Similarly, a rule $\bigcup_{\lambda \in \mathcal{S}} A_{\lambda} \rightarrow \bigcup_{\lambda \in \mathcal{S}} B_{\lambda}$ such that $\bigcup_{\lambda \in \mathcal{S}} A_{\lambda}$ overlap neither $A_{0}$, nor $A_{1}$ is always coherent w.r.t. $\mathcal{K}$. And it can be easily shown that the case 2 of Section II-A (for which $\exists \lambda \in[0,1]$ such that $A^{\star} \supseteq A_{\lambda}$ ) corresponds to the CRI with $A^{\star}$ and such a rule. Thus it is sound to use the proposed method for these two cases, while it is not for the third one.

To summarize, the proposed method is equivalent to the CRI applied to $\mathcal{L}$ in the coherent case (as previously said). In the incoherent case, the method is sound (and still corresponds to the CRI, but only with one well-chosen rule instead of the set $\mathcal{L}$ ) only with input sets $A^{\star}$ greater or equal to one $A_{\lambda}$. Smaller $A^{\star} \mathrm{s}$ should then be approximated by a well-chosen $A_{\lambda}$. A good choice can be the $A_{\lambda}$ centered on $A^{\star}$.

\section{F. Rules with several inputs}

This section briefly deals with the case of rules with several inputs, i.e., the case of a multi-dimensional input universe $U=U_{1} \times \ldots \times U_{p}$. Two main approaches can be considered, depending on the number of rules available for the interpolation process.

First consider that the set of rules $\mathcal{K}$ still contains two rules. The generalization of the method proposed in the previous sections consists in representing $A^{\star}$ by means of $A_{\lambda} \mathrm{s}$, and then computing $B^{\star}$ using the $\lambda \mathrm{s}$. It comes down to the computation, for each dimension $i$, of the conclusion $B_{i}^{\star}$ obtained from the two rules with a mono-dimensional input $A_{0, i} \rightarrow B_{0}$ and $A_{1, i} \rightarrow B_{1}$. And then to the computation of the convex union of the $B_{i}^{\star}$ s. In fact, it is acceptable when the (imprecise) point $A^{\star}$ is on the (imprecise) line defined by the two (imprecise) points $A_{0}$ and $A_{1}$ in the universe $U$, but in the general case it gives very imprecise results.

This is why a better method consists in considering more rules (if available) to define an imprecise hyper-plane of $U \times V$ (as well as the imprecise line defined by $[\underline{\Gamma}(u), \widetilde{\Gamma}(u)]$ depicted on Figure 5 for one input). If $U$ has $p$ dimensions (and $V$ one) it requires $p+1$ rules whose condition parts $A_{i}$ are "around" $A^{\star}$. This method resembles the one described at the end of [7] and in [8]. These two methods are not developed here for the sake of brevity. Note however that they involve some nontrivial problems of classical interpolation in multidimensional spaces.

\section{FUZZY CASE}

In this section, the sets involved in the considered rules may be fuzzy instead of just intervals. The considered fuzzy sets are trapezoïdal-shaped, not only because they are simple to deal with, but ratherbecause they are the most commonly used in practical applications. The extension to more general fuzzy sets is left for further research.

In order to generalize the proposed interpolation method to fuzzy sets, let us first study some properties of the union and intersection of the corresponding $A_{\lambda} \mathrm{s}$ and the $B_{\lambda} \mathrm{s}$. In this case, $A_{\lambda}$ is obtained from $A_{0}$ and $A_{1}$, applying the linear interpolation mechanism of the previous section on each of its $\alpha$-cuts (which are supposed to be intervals): $\left(A_{\lambda}\right)_{\alpha}=$ $\lambda \cdot\left(A_{1}\right)_{\alpha}+(1-\lambda) \cdot\left(A_{0}\right)_{\alpha}$, where $(A)_{\alpha}$ is the $\alpha$-cut of $A$. The following properties are obvious.

- If the fuzzy sets $A_{0}$ and $A_{1}$ are trapezoïdal-shaped, then the fuzzy sets $A_{\lambda}$, for $\lambda \in[0,1]$ are also trapezoïdal-shaped.

Then, it can be shown that this property is also true for the union or intersections of $A_{\lambda}$ s considered by the interpolation mechanism.

- If the fuzzy sets $A_{0}$ and $A_{1}$ are trapezoïdal-shaped, then the union $\bigcup_{\lambda \in I} A_{\lambda}$, where $I \subseteq[0,1]$ is an interval, is also trapezoïdal-shaped.

The requirement for $I$ to be an interval is not always necessary. The union of the cores of the $A_{\lambda}$, for $\lambda \in I$ being an interval is sufficient for the union to be trapezoïdal-shaped. However, as in II-B, the set $I=\mathcal{S}_{\max }$ which is used in the following is an interval.

- If the fuzzy sets $A_{0}$ and $A_{1}$ are trapezoïdal-shaped, then the intersection $\bigcap_{\lambda \in I} A_{\lambda}$, for any $I \subseteq[0,1]$, is also trapezoïdal-shaped, which may be degenerated into a triangle, a sub-normalized triangle, or the empty set.

Although these results give interesting properties on the shapes of unions or intersections of $A_{\lambda} \mathrm{s}$, it is rather clear that, in the general case, a trapezoïdal-shaped input $A^{\star}$ cannot be recovered exactly by such union or intersections. It would require very particular properties on the slopes of $A^{\star}$.

To generalize the interpolation method to the fuzzy case, the next section proposes an approximation of $A^{\star}$ by a union or an intersection of $A_{\lambda}$ s. Then, Section III-B shows that applying the method proposed for intervals to an $\alpha$-cuts decomposition of the fuzzy sets in order to obtain $\alpha$-cuts of $B^{\star}$ correspond to the method proposed by Kóczy and Hirota [2] and thus raises the same problems.

\section{A. Approximation of $A^{\star}$}

Since $A^{\star}$ cannot be exactly retrieved in general, a first solution consists in computing an approximation of $A^{\star}$. However this approximation should be meaningful. Considering the interpretation of fuzzy rules based on possibility theory (see for instance [16]), the conclusion of a rule modeled with a genuine implication is a restriction of the set of possible values for the output variable. Thus, for this restriction imposed by $B^{\star}$ not being arbitrary, its approximation has to be a superset of $B^{\star}$, which means that the approximation of $A^{\star}$ has also to be a superset of $A^{\star}$. Moreover in order not to lose information, this superset has to be the smallest possible one. For a given $A^{\star}$ in between $A_{0}$ and $A_{1}$, such an approximation obtained either by union, or by intersection of $A_{\lambda} \mathrm{s}$ is unique. 
For trapezoïdal-shaped fuzzy sets, the inclusion $A^{\star} \subseteq A_{\lambda}$, defined by $A^{\star} \subseteq A_{\lambda}$ if $\forall u \in U, \mu_{A^{*}}(u) \leq \mu_{A_{\lambda}}(u)$, is equivalent to the classical inclusion of the core and of the support $\left(C\left(A^{\star}\right) \subseteq C\left(A_{\lambda}\right)\right.$ and $\left.S\left(A^{\star}\right) \subseteq S\left(A_{\lambda}\right)\right)$.

As well as in Section II, there are 3 different cases:

1. $A^{\star}=A_{\lambda}$, for one $\lambda \in[0,1]$,

2. $A^{\star} \subset A_{\lambda}$, for one $\lambda \in[0,1]$. In this case $A^{\star}$ is approximated by $\bigcap_{\lambda \in \mathcal{S}_{\max }} A_{\lambda}$ with $\mathcal{S}_{\max }=\left\{\lambda \in[0,1], A^{\star} \subset A_{\lambda}\right\}$. 3. $A^{\star} \nsubseteq A_{\lambda}, \forall \lambda \in[0,1]$ (i.e., all the other cases). Then, $A^{\star}$ is approximated by $\bigcup_{\lambda \in \mathcal{S}_{\max }} A_{\lambda}$, where $\mathcal{S}_{\max }=[\underline{\lambda}, \bar{\lambda}]$ is given by $\underline{\lambda}=\sup _{A^{*} \subseteq\left[A_{\lambda}, A_{1}\right]} \lambda$, (denoting $\left[A_{\lambda}, A_{1}\right]$ the convex hull of $A_{\lambda}$ and $\left.A_{1}\right)$ and $\bar{\lambda}=\inf _{A^{*} \subseteq\left[A_{0}, A_{\lambda}\right]} \lambda$.

Then, considering the interval $\mathcal{S}_{\max }=[\underline{\lambda}, \bar{\lambda}]$, the computation of the trapezoïdal-shaped fuzzy set $B^{\star}$ can be done as in Section II, computing the left and right sides of the trapezoïd. There are also incoherent cases, in particular (but not only) when the obtained $B^{\star}$ is sub-normalized.

\section{B. Computation on the $\alpha$-cut decomposition of $A^{\star}$}

This approach consists in i) decomposing the fuzzy sets into their $\alpha$-cuts, ii) using the method proposed in Section II on the $\alpha$-cuts, iii) and then computing $B^{\star}$, also from its $\alpha$-cuts. This method seems natural since it provides an exact representation of $A^{\star}$. Moreover, computation with level cuts are often used in fuzzy logic, and especially for fuzzy interpolation.

However, it can be shown that it presents the same drawbacks as the method proposed by Kóczy and Hirota [2]. In particular, the computed $\alpha$-cuts of $B^{\star}$ may not fulfill the nestedness requirement. Figure 6 shows an example. Although it corresponds to a coherent case, it shows the relations induced by this method for the support (in light grey) and core (in a darker grey). For the proposed $A^{\star}$, it is immediate that the core and support of the $B^{\star}$ computed this way are disjoint !

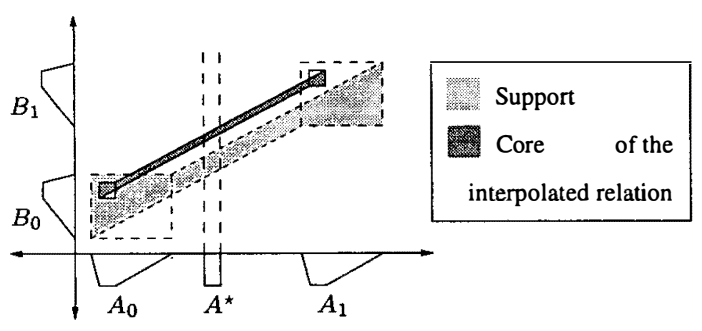

Fig. 6. Interpolation by $\alpha$-cut decomposition

Now, considering that all the fuzzy rules in $\mathcal{K}$ or $\mathcal{L}$ are gradual rules, modeled with Rescher-Gaines implication, the family of rules $\left(A_{\lambda}\right)_{\alpha} \rightarrow\left(B_{\lambda}\right)_{\alpha}, \alpha \in[0,1]$, obtained from one rule by $\alpha$-cutting $A_{\lambda}$ and $B_{\lambda}$, gives an infinity of classical rules whose input and output parts are intervals. Computation with this (double) infinity of rules (or only some of them) and the CRI could lead to an acceptable result, while also processing by $\alpha$-cut decomposition. However, the probability for inconsistency between rules is increased. This is a topic for further research.

\section{CONCLUDING REMARKS}

This paper has outlined a method to interpolate between sparse fuzzy data, which has been also compared to approaches based on the Compositional Rule of Inference. Although this method corresponds to the CRI applied to one rule, defined by union or intersection of linearly interpolated rules, it is different from the CRI applied to the whole set of interpolated rules, which may be incoherent. This lead to a notion of incoherence for sparse rules which ought to be detailed in further developments. Besides, when three rules are considered, $A_{i} \rightarrow B_{i}, i=1, \ldots, 3$, such that $A_{1}$ is in between $A_{0}$ and $A_{2}$, taking $A^{\star}=A_{1}$ is a possible way to check whether or not this set of 3 rules is compatible with the linear interpolation principle in the sense that $B^{\star} \supseteq B_{1}$. If $B^{\star} \subseteq B_{1}$, removing the rule $A_{1} \rightarrow B_{1}$ could be thought of, since the interpolation provides a more precise result. Otherwise, and in particular if $B^{\star} \cap B_{1}=\emptyset$, the interpolation should only be done between $A_{0}$ and $A_{1}$, or $A_{1}$ and $A_{2}$, and not between $A_{0}$ and $A_{2}$.

\section{REFERENCES}

[1] L.A. Zadeh, "Outline of a new approach to the analysis of complex systems and decision processes," IEEE Transactions on Systems, Man and Cybernetics, vol. 3, pp. 28-44, 1973.

[2] L.T. Kóczy and K. Hirota, "Interpolative reasoning with insufficient evidence in sparse fuzzy rule bases," Information Sciences, vol. 71, pp. 169-201, 1993.

[3] L.T. Kóczy and K. Hirota, "Ordering, distance and closeness of fuzzy sets," Fuzzy Sets and Systems, vol. 59, pp. 281-293, 1993.

[4] S. Yan, M. Mizumoto, and W.Z. Qiao, "Reasoning conditions on Kóczy's interpolative reasoning method in sparse fuzzy rule bases," Fuzzy Sets and Systems, vol. 75, no. 1, pp. 63-71, 1995.

[5] W.Z. Qiao, M. Mizumoto, and S. Yan, "An improvement to Kóczy and Hirota's interpolative reasoning in sparse fuzzy rule bases," International Joumal of Approximate Reasoning, vol. 15, pp. 185-201, 1996.

[6] W.H. Hsiao, S.M. Chen, and C.H. Lee, "A new interpolative reasoning method in sparse rule-based systems," Fuzzy Sets and Systems, vol. 93. no. 1 , pp. 17-22, 1998

[7] D. Dubois, H. Prade, and L. Ughetto "Fuzzy logic, control engineering and artificial intelligence," in Fuzzy Algorithms for Control Engineering. H.B. Verbruggen, H.-J. Zimmermann, and R. Babuska, Eds., pp. 17-57. Kluwer Academic Publishers, 1999.

[8] D. Dubois and H. Prade, "On fuzzy interpolative reasoning," Int. Journal of General Systems, vol. 28, pp. 103-114, 1999.

[9] B. Bouchon-Meunier, J. Delechamp, C. Marsala, N. Mellouli, M. Rifqi, and L. Zerrouki, "Analogy and fuzzy interpolation in case of sparse rules," in Proc. of the EUROFUSE-SIC Joint Conf., 1999, pp. 132-136.

[10] B. Bouchon-Meunier, J. Delechamp, C. Marsala, N. Mellouli, M. Rifqi and L. Zerrouki, "Raisonnement interpolatif à partir de schéma analogique flou," in e-proc. of Jour. Nat. (JNMR'99), Paris, France, 1999.

[11] B. Bouchon-Meunier, J. Delechamp, C. Marsala, and M. Rifai, "Several forms of fuzzy analogical reasoning," in Proc. of the 6th IEEE Int. Conf on Fuzzy Systems (FUZZ-IEEE'97), 1997, vol. 1, pp. 45-50.

[12] D. Dubois, R. Martin-Clouaire, and H. Prade, "Practical computing in fuzzy logic," in Fuzzy Computing, M.M. Gupta and T. Yamakawa, Eds., pp. 11-34. North-Holland, 1988.

[13] JC. Buisson, TOULMED, un générateur de systèmes-experts qui prend en compte l'imprécision et l'incertitude des connaissances, $\mathrm{Ph} . \mathrm{D}$. thesis, Univ. Paul Sabatier, Toulouse III, France, 1987, pp. 129-139.

[14] D. Dubois, H. Prade, and L. Ughetto, "Checking the coherence and redundancy of fuzzy knowledge bases," IEEE Transactions on Fuzzy Systems, vol. 5, no. 3, pp. 398-417, Aug. 1997.

[15] D. Dubois, M. Grabisch, and H. Prade, "Gradual rules and the approximation of control laws," in Theoretical Aspects of Fuzzy Control, $\mathrm{H}$ Nguyen, M. Sugeno, R. Tong, R. Yager, Eds., pp. 147-181. Wiley, 1994.

[16] L. Ughetto, D. Dubois, and H. Prade, "Implicative and conjunctive fuzzy rules - A tool for reasoning from knowledge and examples," in Proc. of the 16th American Nat. Conf. on AI (AAAI'99), 1999, pp. 214-219. 